\title{
Identification and management of the high risk patient with dilated cardiomyopathy
}

\author{
M Borggrefe, M Block, G Breithardt
}

Congestive heart failure is a common cause of death in Europe and the United States. Depending on the severity of the disease, annual mortality ranges from $10 \%$ to $50 \% .^{1-4}$ The mode of death is circulatory failure or sudden cardiac death, which accounts for approximately $50 \%$ of deaths. ${ }^{1-4}$ The proportion of sudden death in various studies ranges from $4 \%$ to $90 \%$. This variation may be explained partly by different definitions, different patient populations, and the inclusion of non-classifiable deaths in the sudden death category. Non-ischaemic (idiopathic) dilated cardiomyopathy (DCM) is the most common aetiology. In some reports, however, no attempt was made to distinguish between non-ischaemic and ischaemic left ventricular dysfunction because only non-invasive diagnostic procedures were performed. A major shortcoming of some large prospective studies of patients with heart failure is their inclusion of two completely different subgroups (ischaemic and non-ischaemic left ventricular dysfunction). There are many clinical and haemodynamic variables that might predict a poor prognosis owing to impending circulatory failure. It is difficult to identify the patient at risk of sudden death, however, because there are so many different mechanisms and changes in left ventricular function.

\section{Mechanisms of sudden death in DCM}

Despite the lack of data on the mechanisms of sudden death in patients with DCM, it has been assumed that primary ventricular fibrillation or ventricular tachycardia degenerating into ventricular fibrillation are the most common modes of death (table 1). In addition, asystole was the primary electrical event in some patients with DCM. There is postmortem evidence that an embolic cerebral event or even pulmonary embolism can cause sudden death, especially in patients with severe biventricular heart failure. Recently Luu et al reviewed the causes of cardiac arrest in advanced heart failure in 20 patients (13 with ischaemic cardiomyopathy and seven with non-ischaemic DCM). ${ }^{5}$ In 13 patients $(62 \%)$ the rhythm at the time of arrest was severe bradycardia or electromechanical dissociation. Only $38 \%$ of arrests were caused by ventricular tachycardia or fibrillation. This incidence of ventricular tachyarrhythmias is significantly lower than might be expected from studies of all patients with cardiac arrest.
The extent to which treatment with an antiarrhythmic drug facilitates the development of bradyarrhythmias or electromechanical dissociation is not known. Because sudden death in DCM is multifactorial, risk stratification and management of patients is extremely difficult.

\section{Risk stratification in DCM}

Mortality in DCM is closely related to left ventricular function as indexed by high ventricular filling pressures, high end diastolic and end systolic volumes, and low ventricular ejection fraction. ${ }^{12}$ Many studies suggest that the left ventricular ejection fraction is the strongest predictor of death. However, although ejection fraction is a predictor of total deaths, it may not be the best predictor of arrhythmic death in DCM because half these deaths are sudden. Moreover, some reports suggest that a higher proportion of patients in functional class I and II die suddenly than patients in functional class III or IV in whom progressive heart failure usually causes death. $^{2}$

The role of asymptomatic arrhythmias detected during Holter monitoring in the prediction of sudden death is controversial. Atrial fibrillation is common in patients with advanced heart failure $(9-35 \%){ }^{34}$ In the Veterans Administration Cooperative Trial atrial fibrillation detected during Holter monitoring was not related to mortality. ${ }^{3}$ However, Middlekauff $e t$ al recently examined the relation of atrial fibrillation to prognosis in 390 patients with advanced heart failure. ${ }^{4}$ They found that atrial fibrillation was associated with an increase in total mortality and in the rate of sudden death. In most studies nonsustained ventricular tachycardia during Holter monitoring has not been found to worsen prognosis independently. ${ }^{67}$ Meinertz et $a l$ and von Olshausen $e t$ al studied the incidence and prognostic significance of ventricular arrhythmias in patients with idiopathic DCM. ${ }^{89}$ Both studies found that the risk of death was high in patients with poor left ventricular function and frequent non-sustained ventricular tachycardia. Although these studies suggest that asymptomatic, complex ventricular arrhythmias are of prognostic importance other invèstigators did not find a similar relation. The sensitivity of Holter monitoring in detecting frequent ventricular ectopy or non-sustained ventricular tachycardia seems to be high, but its predictive value with regard to sudden death seems to be low. 
The presence of ventricular late potentials on the signal averaged electrocardiogram was associated with a poor prognosis in patients with ischaemic heart disease. Several studies that evaluated the use of signal-averaged electrocardiography in the assessment of individuals with diverse underlying clinical diagnosis included a few patients with non-ischaemic DCM. Several small studies suggested that the signal averaged electrocardiogram was useful in the prediction of malignant ventricular tachyarrhythmias in patients with DCM. In patients with coronary artery disease an abnormal signal averaged electrocardiogram successfully identified patients with a high propensity for sustained ventricular tachycardia or even sudden cardiac death. The data were not as conclusive in patients with DCM.

Recently, Mancini et al reported on 114 consecutive patients referred for evaluation for heart transplantation with non-ischaemic DCM. ${ }^{10}$ They evaluated the usefulness of signal averaged electrocardiography and the impact of bundle branch block QRS morphology on the development of malignant arrhythmic events and all-cause mortality. The best predictor of an adverse outcome defined as death, transplantation, or occurrence of sustained ventricular tachycardia was a higher New York Heart Association classification. The only other predictor of an adverse outcome was an abnormal signal averaged electrocardiogram. These are promising results. However, the clinical application of signal averaged electrocardiography in patients with DCM has the same limitations as those inherent in its use in patients after myocardial infarction. The negative and positive predictive values for predicting sudden death or sustained ventricular tachycardia were $100 \%$ and $45 \%$, respectively. Thus when interventions are considered in high risk patients many patients may be treated unnecessarily. None the less, as in post myocardial infarction patients, the prognosis is good if the signal averaged electrocardiogram is normal. Currently, the value of this non-invasive technique for risk stratification of patients with DCM is presently not known because there have been no prospective, large studies in mildly symptomatic patients.

There are studies of the prognostic value of programmed ventricular stimulation in symptom free patients with DCM. ${ }^{112}$ Sustained ventricular tachycardia or fibrillation was inducible in up to $40 \%$ of patients (table 2 ). These studies showed that the ability of programmed ventricular stimulation to identify those patients with DCM at risk of sudden death was limited because:

Table 2 Role of programmed ventricular stimulation in risk stratification of symptom free patients with DCM

\begin{tabular}{lll}
\hline & Patients (n) & Induced VT/VF (\%) \\
\hline Meinertz, et al 1985'12 & 42 & $2 \cdot 4$ \\
Stamato 1986 & 15 & 0 \\
Das 1986 & 24 & 40 \\
Stevenson, et al 1988 & 72 & 13 \\
\hline
\end{tabular}

VT, ventricular tachycardia; VF, ventricular fibrillation
The yield of inducible monomorphic ventricular tachycardia was low

- Intense stimulation techniques lead to polymorphic ventricular tachycardia or ventricular tachycardia that was unspecific.

Clearly, risk stratification of patients with DCM is extremely difficult. Poor left ventricular function seems to be the strongest predictor of total cardiac mortality. The prediction of sudden death in patients with DCM is poor.

\section{Antiarrhythmic drug treatment in symptom free patients with DCM and non-sustained ventricular tachycardia} Neither class I antiarrhythmic drugs nor amiodarone reduced total mortality or sudden death in patients with DCM and non-sustained ventricular tachycardia. Furthermore, the risks of antiarrhythmic treatment are higher in patients with reduced left ventricular function: arrhythmogenic effects are more likely in these patients and heart failure is exacerbated in $4-25 \%$ of patients, especially when class I drugs are used. Because the role of asymptomatic, complex ventricular arrhythmias in determining prognosis in DCM is not known and the therapeutic end point is not clear, it is probably prudent to avoid treatment with antiarrhythmic drugs in this group of patients. We cannot be certain that non-pharmacological interventions such as the implantation of a cardioverterdefibrillator in asymptomatic patients with DCM are beneficial until the results of properly designed, prospective studies such as the German Dilative Cardiomyopathy Study, which has recently been started, are known. Until then treatment of arrhythmias in DCM should be directed towards eliminating arrhythmogenic factors-that is, hypokalaemia, hypomagnesaemia, activation of the sympathetic nervous system and the reninangiotensin-aldosterone system, and the arrhythmogenic effects of inotropic agents. Treatment would therefore include potassium sparing diuretics and potassium and magnesium supplementation and the careful use and titration of $\beta$ blockers, angiotensin converting enzyme inhibitors, digitalis, and inotropic agents. Programmed ventricular stimulation may be indicated in a subgroup of patients with DCM and long runs of nonsustained ventricular tachycardia. Electropharmacologically guided treatment may be useful in some of these patients. So far, however, there are no prospective studies on this aspect of treatment.

\section{Management of patients with sustained ventricular tachycardia and/or ventricular fibrillation in DCM}

Patients with sustained ventricular tachycardia or out of hospital ventricular fibrillation and DCM are at high risk of recurrent, lifethreatening tachyarrhythmias and sudden cardiac death. ${ }^{13-16}$ Several large studies have validated the use of electropharmacological 
Table 3 Inducibility of sustained ventricular tachycardia/ventricular fibrillation (VT/VF) in patients with documented, sustained ventricular tachyarrhythmias

\begin{tabular}{|c|c|c|c|}
\hline Study & Patients (n) & Inducible VT/VF (n(\%)) & Sudden death (n (\%)) \\
\hline $\begin{array}{l}\text { Pol et al } 1984^{13} \\
\text { Brembilla-Perrot et al } 1991^{14} \\
\text { Constantin et al } 1989^{15} \\
\text { Liem and Swerdlow } 1988^{17} \\
\text { Milner et al } 1988^{16} \\
\text { Rae et al } 1987^{18} \\
\text { Borggrefe } \\
\text { Total }\end{array}$ & $\begin{array}{l}11 \\
11 \\
27 \\
64 \\
19 \\
38 \\
98 \\
\mathrm{n}=268\end{array}$ & $\begin{array}{l}11(100) \\
8(73) \\
14(52) \\
43(52) \\
13(68) \\
25(66) \\
54(55) \\
\mathrm{n}=168(63 \%)\end{array}$ & $\begin{array}{l}4 / 11(36) \\
3 / 8(37) \\
2 / 14(14) \\
\text { NA } \\
\text { NA } \\
2 / 25(8) \\
4 / 54(7)\end{array}$ \\
\hline
\end{tabular}

NA not available lation at baseline or poor reproducibility of ventricular tachycardia induction, serial drug testing is not helpful in many patients. Several studies have shown a high recurrence rate for ventricular tachycardia or fibrillation or even sudden death in those patients in whom arrhythmia was not inducible at baseline. ${ }^{1719}$ These findings show that serial drug testing in patients with DCM is useful only in those in whom ventricular tachycardia or fibrillation can reproducibly be induced at baseline when antiarrhythmic drugs are not being giventhat is, for about $50-56 \%$ of patients with DCM and a history of sustained ventricular tachycardia or fibrillation. However, a favourable drug response (that is, suppression of inducibility of ventricular tachycardia) can only be obtained in about $30 \%$ of patients. ${ }^{17}$ Therefore, non-pharmacological therapy such as catheter ablation or implantation of a cardioverter-defibrillator should be considered early in the management of patients with DCM and life-threatening ventricular tachyarrhythmias. ${ }^{1920}$ In the absence of prospective, randomised trials to compare serial drug testing and implantation of a cardioverterdefibrillator we suggest the following approach (figure) to treatment of patients with DCM and sustained ventricular tachycardia or fibrillation who are not primary candidates for heart transplantation. If sustained ventricular tachycardia or fibrillation cannot be induced or cannot be reproducibly induced during baseline electrophysiological evaluation a cardioverter-defibrillator should be implanted. If sustained ventricular tachycardia or fibrillation is reproducibly induced patients should have serial drug testing. In about $30-50 \%$ of patients arrhythmia can be controlled by antiarrhythmic drugs. If no drug is effective, patients who, judged by their left ventricular dysfunction, are expected to live at least for a year should be offered a cardioverter-defibrillator. A retrospective analysis of our own series of patients with DCM undergoing programmed electrical stimulation showed that the availability of the implantable cardioverter-defibrillator (since 1984 in Germany) considerably reduced the risk of sudden death in patients with DCM. ${ }^{19}$

Between 1979 and 1987, 84 patients needed treatment because of documented or suspected ventricular tachycardia or fibrillation (ICD implantation rate $5 \%$ ). This group of patients was compared with a cohort studied between 1988 and 1991 ( $n=80$ patients, ICD implantation rate $39 \%$ ). Eleven per cent of patients died suddenly in the early period (1979 to 1987 ) compared with $2.5 \%$ in the later period (1988 to 1991). The follow up periods in the two cohorts are not comparable, nevertheless, most arrhythmic events occurred during the first year after the initial electrophysiological evaluation. Therefore, these results of this non-randomised, retrospective study show that the availability of an implantable cardioverter-defibrillator considerably improved outcome. Recently, Fazio $e$ al analysed the long-term outcome of 40 patients with DCM and ventricular tachy- 
cardia or fibrillation undergoing implantation of a cardioverter-defibrillator. ${ }^{20}$ The actuarial mortality at 1 and 4 years was $0 \%$ and $14 \%$ for sudden death. Compared with projected mortality these results confirm a significant improvement in prognosis of patients with DCM and life-threatening ventricular tachyarrhythmias and are consistent with similar analyses by other workers in patients with ischaemic heart disease undergoing implantation of a cardioverter-defibrillator.

1 Massie B, Ports T, Chatteriee $\mathrm{K}$, et al Long-term vasodilator therapy for heart failure: clinical response and its
relationship to hemodynamic measurements. Circulation relationship to hem.

2 Wilson JR, Schwartz JS, St John Sutton M, et al. Prognosis in severe heart failure: relation to hemodynamic measurements and ventricular ectopic activity. $\mathcal{F} \mathrm{Am}$ Coll Card 1983;2:403-9.

3 Carson P. Atrial fibrillation/flutter does not decrease survival in congestive heart failure [abstr]. $₹ \mathrm{Am} \mathrm{Coll}$ Cardiol 1991;17:90.

4 Middlekauff HR, Stevenson WG, Stevenson LW. Prognostic significance of atrial fibrillation in advanced heart failure. Circulation 1991;84:40-8.

5 Luu M, Stevenson WG, Stevenson LW, et al. Diverse mechanisms of unexpected cardiac arrest in advanced heart failure. Circulation 1989;80:1675-80.

6 Franciosa JA, Wilen M, Ziesche S, et al. Survival in men with severe chronic left ventricular failure due to either with severe chronic left ventricular failure due to either
coronary heart disease or idiopathic dilated cardiomycoronary heart disease or idiopathic

7 Huang SK, Messer JV, Denes P. Significance of ventricular tachycardia in idiopathic dilated cardiomyopathy: observation in 35 patients. Am $\mathcal{F}$ Cardiol 1983;51:507-12.

8 Meinertz T, Hofmann T, Kasper W, et al. Significance of ventricular arrhythmias in idiopathic dilated cardiomyopathy. Am $\mathcal{F}$ Cardiol 1984;53:902-7.

9 von Olshausen K, Schäfer A, Mehmel HC, Schwarz F, Senges J, Kübler $W$. Ventricular arrhythmias in idio- pathic dilated cardiomyopathy. $\mathrm{Br}$ Heart $\mathcal{f}$ 1984;51: 195-201.

10 Mancini DM, Wong KL, Simson MB. Prognostic value of an abnormal signal-averaged electrocardiogram in patients with nonischemic congestive cardiomyopathy. patients with nonischemic

11 Stevenson WG, Stevenson LW, Weiss J, et al. Inducible ventricular arrhythmias and sudden death during vasodilator therapy of severe heart failure. Am Heart $\mathcal{F}$ 1988;116:1447-54

12 Meinertz T, Treese N, Kasper W, et al. Determinants of prognosis in idiopathic dilated cardiomyopathy as determined by programmed electrical stimulation. $\mathrm{Am}$ Cardiol 1985;56:337-41.

13 Poll DS, Marchlinkski FE, Buxton AE, et al. Sustained ventricular tachycardia in patients with idiopathic dilated cardiomyopathy: electrophysiologic testing and lack of response to antiarrhythmic drug therapy. Circulation 1984;70:451-6.

14 Brembilla-Perrot B, Donetri J, Terrier de la Chaise A, et al. Diagnostic value of ventricular stimulation in patients Diagnostic value of ventricular stimulation in patients
with idiopathic dilated cardiomyopathy. Am Heart $\mathcal{f}$ 1991;121:1124-31.

15 Constantin L, Martins JB, Kienzle MG, et al. Induced sustained ventricular tachycardia in nonischemic dilated cardiomyopathy: dependence on clinical presentation and response to antiarrhythmic agents. $P A C E$ 1989;12: 776-83.

16 Milner PG, Dimarco JP, Lerman BB, et al. Electrophysiological evaluation of sustained ventricular tachyarrhythmias in idiopathic dilated cardiomyopathy. PACE 1988;11:562-8.

17 Liem LB, Swerdlow CD. Value of electropharmacologic testing in idiopathic dilated cardiomyopathy and sustained ventricular tachyarrhythmias. Am 7 Cardiol 1988;62:611-6.

18 Rae AP, Spielman SR, Kutalek SP, et al. Electrophysiologic assessment of antiarrhythmic drug Electrophysiologic assessment of antiarrhythmic drug dilated cardiomyopathy. $A m \ngtr$ Cardiol 1987;59:291-5.

19 Borggrefe M, Chen X, Shenasa M, Breithardt G. Clinical value of programmed ventricular stimulation in dilative cardiomyopathy: acute and long-term results. Unpublished.

20 Fazio G, Veltri EP, Tomaselli G, et al. Long-term follow-up of patients with nonischemic dilated cardiomyopath and ventricular tachyarrhythmias treated with implantable cardioverter defibrillators. PACE 1991;14: 1905-10. 